\title{
Perceptions de stagiaires quant au soutien des deux formateurs à l'articulation entre théorie et pratique
}

Josianne Caron

Université du Québec à Trois-Rivières

(Canada)

Liliane Portelance

Université du Québec à Trois-Rivières

(Canada)

\author{
Student teachers' perceptions in relation \\ to the support provided by both trainers regarding \\ the articulation between theory and practice
}

\section{ésumé}

En formation à l'enseignement, les étudiants sont appelés à articuler des savoirs théoriques et pratiques. Or, les stagiaires peinent à percevoir l'interdépendance des savoirs et ont besoin d'être soutenus pour y parvenir (Waege et Haugalokken, 2013). Quel soutien obtiennentils de leurs formateurs? Six stagiaires québécois en stage d'internat au secondaire et leurs formateurs, l'enseignant associé et le superviseur universitaire, ont participé à une recherche interprétative. Une entrevue individuelle avec les stagiaires a porté sur le soutien perçu. L'analyse révèle que les stratégies de soutien à l'articulation entre théorie et pratique, quoique prédominantes chez le superviseur,

sont distinctes et complémentaires. Mots-clés Formation à l'enseignement, stages en enseignement secondaire, articulation théorie-pratique, formation en alternance, formateurs du stagiaire

Abstract

In teaching training, students are invited to articulate the theoretical and practical knowledge. Yet, the trainees have difficulty in perceiving and understanding the interdependence of the knowledge. They especially need support to achieve it (Waege \& Haugalokken, 2013). What support is then brought by the trainer to the trainee? Six trainees in internship in the secondary sector and their trainers, in various regions from Quebec, participated in an interpretative research. An individual

interview with the trainees concerned the perceived support. The data analysis reveals that the strategies of support for the articulation between theory and practice, although dominant at the supervisor, are different and complementary.

\section{Keywords}

Teacher training, teaching internship in the secondary sector, articulation between theory and practice, student teacher trainers, alternating training

\section{Mise en contexte}

Au Québec,la formationinitialeàl'enseignement estorientée, selon une approche-programme, vers la professionnalisation de l'enseignement (Ministère de l'Éducation du Québec, 2001). Cette orientation invite le futur professionnel à une appropriation et une intégration réfléchies de connaissances théoriques et de connaissances issues de la pratique (Malglaive, 1994). Dans l'optique d'une articulation entre la théorie et la pratique, elle exige le développement de la capacité à mettre en question les connaissances théoriques en prenant en considération le contexte authentique de travail. Elle sollicite également l'aptitude à analyser les situations éducatives réelles à la lumière de connaissances issues de la recherche. L'étudiant est incité à exercer un jugement critique sur les connaissances de sa formation afin que son agir professionnel s'appuie sur des choix fondés.

En vue de stimuler son habileté à s'interroger sur ses acquis et son interprétation de la réalité, le futur enseignant québécois progresse dans un parcours de formation en alternance d'une durée de quatre années au cours desquelles il reçoit 700 heures de formation en milieu de pratique. Dans ce milieu, il bénéficie de l'encadrement de deux formateurs : l'enseignant associé et le superviseur universitaire. Ces derniers sont invités à travailler en collaboration (Johnson et Napper-Owen, 2011; Portelance, Martineau et Caron, sous presse), et ce, bien que des facteurs contextuels n'y soient pas toujours favorables. Mentionnons que les rencontres des deux formateurs du stagiaire sont peu fréquentes, l'enseignant associé étant continuellement présent dans son école auprès du stagiaire et le superviseur ayant comme fonction de superviser plusieurs stagiaires dans plusieurs lieux distincts et éloignés. Généralement, les seules rencontres en présentiel ont lieu lors des visites de supervision au cours desquelles la triade se réunit après la période d'observation en classe. D'autres échanges se déroulent par courriel ou par téléphone. 
Lorsque le stagiaire vit une immersion dans le milieu scolaire, il est appelé à percevoir l'interdépendance, la complémentarité et l'articulation de la théorie et de la pratique. Or, il demande de l'aide puisque, pour lui, cette articulation est difficile (Waege et Haugalokken, 2013). Comment perçoit-il le soutien qu'il obtient de ses formateurs? Ce texte vise à faire état de constats sur cet objet d'étude. Avant de poser quelques assises théoriques et des repères méthodologiques, nous situons le problème de la difficile articulation de la théorie et de la pratique. Le texte est complété par une présentation des principaux résultats et de leur interprétation.

\section{Éléments de problématique}

Le dispositif de formation initiale selon lequel le futur enseignant s'approprie au premier chef des connaissances abordées dans les cours théoriques afin de les appliquer ultérieurement dans la pratique est de plus en plus discrédité (Tardif et al., 2001). Depuis 2006, un groupe international de recherche sur l'apprentissage en alternance s'intéresse aux défis de l'alternance et soutient qu'il importe de mettre en évidence la nécessité de la circulation bidirectionnelle des savoirs théoriques et pratiques (Portelance et Van Nieuwenhoven, 2010). Dans cette optique, les universités tentent de favoriser l'apprentissage en alternance intégrative pour susciter à la fois le réinvestissement, dans les cours universitaires, des apprentissages réalisés en stage et la mobilisation des apprentissages théoriques ainsi que leur confrontation aux réalités du travail enseignant pendant les stages (Chaubet et Gervais, 2014; Pentecouteau, 2012). Malgré cela, le contexte d'une formation professionnalisante qui se déroule en deux contextes distincts donne difficilement lieu à une alternance intégrative, idéalement caractérisée par une interdépendance effective des deux milieux de formation et des connaissances qu'ils véhiculent respectivement (Merhan, Ronveaux et Vanhulle, 2007).

\section{La difficile articulation entre la théorie et la pratique}

Les recherches sur la formation initiale des enseignants, menées dans différents pays, révèlent les difficultés des étudiants à articuler les connaissances théoriques et pratiques (Waege et Haugalokken, 2013). En effet, ces derniers considèrent que la théorie et la pratique sont incompatibles ou qu'elles appartiennent à des mondes différents. Les mêmes auteurs soutiennent que cette représentation peut avoir un effet négatif sur le développement professionnel des futurs enseignants, ce développement dépendant en partie de la capacité à analyser la pratique enseignante à la lumière de connaissances théoriques.

Un fait semble indubitable : le stagiaire réussit difficilement à donner du sens à la théorie (Portelance et Legendre, 2001), à mobiliser ses connaissances théoriques et à les mettre en relation avec la pratique enseignante (Stürmer, Könings et Seidel, 2013). Ce problème est aussi signalé par les formateurs du stagiaire. Plusieurs chercheurs l'ont constaté (Grossman, Smagorinsky et Valencia, 1999; Stürmer et al., 2013). Confronté à cette difficulté et à la nécessité de concilier les connaissances véhiculées par les milieux scolaire et universitaire, le stagiaire réclame du soutien de ses formateurs (Desbiens, Borges et Spallanzani, 2012). Ce soutien pourrait façonner le processus de construction des significations, lequel est fortement médiatisé par l'enseignant associé et le superviseur dans le discours autour de l'activité professionnelle du stagiaire. La médiation se manifesterait, notamment, par un encadrement qui favorise 
le développement de la compétence à repérer, mobiliser et mettre en relation des connaissances issues de sources variées, les réseaux pédagogiques et la littérature de recherche (Ministère de l'Éducation du Québec, 2001).

\section{Des attentes envers les deux formateurs du stagiaire}

L'encadrement du stagiaire dans une visée d'articulation entre la théorie et la pratique ne peut s'accommoder d'un rapport à la connaissance centré exclusivement sur la théorie ou encore sur la pratique. Quoique nécessaires et généralement fort valables, les connaissances issues de la pratique ne suffisent pas pour faire des choix d'interventions pédagogiques et didactiques fondés (Altet, 2012; Wentzel, 2012). Les connaissances théoriques sont également essentielles, dans la mesure où elles sont soumises à l'épreuve de l'expérience (Beckers, 2007; Malo, 2005). Le terrain offre ainsi un contexte d'innovation et d'émulation de la réflexion théorique. Durant le stage, le stagiaire est d'ailleurs appelé à mobiliser ses savoirs théoriques et à les soumettre à la réalité afin, notamment, d'en estimer les limites ou les lacunes (Perrenoud, 2001b). Il est conséquemment souhaitable que ses deux formateurs l'invitent à les mobiliser consciemment.

Cette attente à l'égard de l'enseignant associé et du superviseur universitaire n'est pas simple à satisfaire. D'une part, les enseignants associés ne sont pas forcément aptes à mobiliser des repères scientifiques (L'Hostie, Monney et Nadeau-Tremblay, 2013) et, d'autre part, les superviseurs peuvent ressentir des besoins de formation théorique (Jacques, 2007). Pourtant, afin de superviser la planification de l'enseignement et le pilotage d'activités d'enseignement du stagiaire en soutenant l'articulation entre la théorie et la pratique, l'enseignant associé et le superviseur ont besoin d'un bagage de connaissances formelles spécialisées issues des sciences humaines et sociales (Perrenoud, 2001a), des sciences cognitives (Brien, 1997) et des sciences de l'éducation (Schneuwly, 2012).

Depuis 2008, un cadre de référence de la formation des formateurs de stagiaires au Québec comporte les compétences attendues respectivement de l'enseignant associé et du superviseur (Portelance, Gervais, Lessard, Beaulieu et collaborateurs, 2008). En particulier, une compétence en matière de pratique réflexive et d'analyse des pratiques enseignantes requiert une approche intégrative de la formation du stagiaire. Cette approche est actualisée dans l'interdépendance, la complémentarité et le statut égalitaire des connaissances théoriques et pratiques. Selon cette posture, les formateurs et les stagiaires repèrent et s'approprient avec discernement les connaissances théoriques et sont en mesure d'ajuster leurs interventions en fonction de ces dernières (Altet, Paquay et Perrenoud, 2002) et de leur pertinence dans un contexte donné. Inversement, ils ont à mettre en mots la pratique enseignante, à en dégager des connaissances expérientielles et à les mettre en relation avec un bagage théorique complémentaire. Or, rappelons-le, les stagiaires peinent à percevoir l'interdépendance des connaissances à articuler (Stürmer et al., 2013). Malgré cela, il arrive fréquemment que l'articulation entre la théorie et la pratique soit laissée aux étudiants eux-mêmes (Borges, 2006).

Face aux difficultés du stagiaire, les formateurs, enseignants associés et superviseurs, sont appelés à agir comme des médiateurs entre la théorie et la pratique. À l'heure actuelle, nous ignorons comment cette attente est prise en compte par ces deux formateurs. Que constatent les stagiaires à ce sujet? Mentionnons que les deux formateurs sont appelés à travailler en concertation et en collaboration 
auprès du stagiaire, et ce, au moyen d'échanges pendant le stage et lors des visites de supervision. Dans le cadre d'une recherche dont l'objectif principal était d'analyser la collaboration entre les deux formateurs du stagiaire, nous avons tenté de répondre à la question suivante : comment des enseignants associés et des superviseurs aident-ils respectivement et conjointement les stagiaires à établir des liens entre leurs savoirs théoriques et pratiques? Le point de vue des stagiaires a été recueilli et analysé. Avant un bref exposé du processus de recherche, nous présentons des référents conceptuels qui aident à mieux saisir la question traitée.

\section{Référents conceptuels}

Dans les paragraphes suivants, nous explorons la relation entre les deux types de savoirs, théoriques et pratiques. Nous voyons ensuite en quoi peut consister le soutien des formateurs du stagiaire à l'articulation entre la théorie et la pratique.

\section{La relation entre théorie et pratique}

Beckers (2007) définit les savoirs théoriques comme des savoirs objectivés et destinés à favoriser la compréhension de l'action. La pratique fait référence à l'agir professionnel, aux activités pédagogiques coutumières, aux façons d'intervenir. Elle reflète l'adaptation au contexte, mais aussi les croyances, les valeurs et les théories personnelles propres à chaque praticien (Malo, 2000). Étant donné ces distinctions, une relation d'opposition entre théorie et pratique peut sembler évidente. Le défi consiste à transformer cette opposition apparente en une articulation, comme le mentionne Altet (2010).

L'articulation de la théorie et de la pratique nécessite pour un stagiaire le recours à des connaissances formellement reconnues pour orienter et analyser son agir professionnel, mais aussi à des expériences concrètes pour mettre à l'épreuve des théories qu'il tente d'actualiser. Elle correspond à un passage de catégories intuitives de lecture des pratiques, de descriptions de sens commun, de prescriptions non interrogées à des interprétations fondées, appuyées sur des résultats argumentés qui permettent de lire des situations afin de nourrir le jugement professionnel (Altet, 2012). Inversement, elle correspond à une lecture expérientielle et critique des référents théoriques de la formation à l'enseignement. Au cœur de cette circulation bidirectionnelle, les savoirs théoriques et pratiques ne sont pas statiques, ils évoluent dans un système dynamique. Pentecouteau (2012) affirme à ce sujet que, dans un dispositif d'alternance-articulation, le «temps formatif, qui se compose dans deux espaces, en interaction l'un avec l'autre » (p. 5), permet le décloisonnement idéal entre savoirs théoriques et savoirs pratiques pour accéder à un savoir professionnel.

Dans une approche critique de l'alternance, Malglaive et Weber (1982) analysent la relation entre théorie et pratique. Deux approches de l'apprentissage, la voie symbolique et la voie matérielle, les incitent à nuancer cette opposition pour mettre l'accent sur la complémentarité. Par la voie symbolique, un apprenant accède aux savoirs théoriques sur le réel et aux procédures d'action sur le réel. Par la voie matérielle, il est confronté au monde matériel et social. Le contact direct s'accompagne de conceptualisations et d'attributions de significations. Or, celles-ci restent limitées aux impératifs de l'action et risquent de ne donner qu'une connaissance approximative ou déformée du réel «si leur élaboration ne s'enrichit pas de procédures et résultats fournis par la théorie » (p. 27). Dans le même 
sens, Beckers (2007) explicite l'articulation entre théorie et pratique en insistant sur le rapport de fonctionnalité réciproque. Selon cette perspective, la théorie sert à élaborer et réguler les processus d'enseignement, alors que la pratique aide à contextualiser, éprouver et réorganiser les contenus théoriques. Dans un dialogue virtuel entre "les deux apparentes solitudes ", la théorie permet d'anticiper la pratique et "l'expérience appelle la théorie à la rescousse de la compréhension ou de l'action " (Chaubet et Gervais, 2014, p. 154). Pour le stagiaire, cette articulation dialogique devient possible et probable si elle prend forme dans une responsabilité partagée par les deux formateurs du stagiaire (Portelance et al., 2008).

\section{Le soutien des formateurs à l'articulation entre théorie et pratique}

Le pari des formateurs du stagiaire, l'enseignant associé du milieu scolaire et le superviseur du milieu universitaire, est d'aider le futur enseignant à donner sens à ses expériences de terrain en tissant des liens étroits avec les théories, tout en mettant la pratique au centre de sa formation (Borges, 2006). Cela nécessite de rendre la théorie significative et vivante et de faire appel au rôle de médiateur du formateur.

Selon Altet (2010), le formateur apprend au stagiaire à analyser les situations éducatives. Dans ce rôle, il est appelé à soutenir le stagiaire dans la mobilisation de ses ressources ainsi que dans l'élaboration et la régulation de savoirs d'action liés à des savoirs de recherche. Il est de plus incité à favoriser les choix personnels issus d'un raisonnement éclairé (Portelance, 2010). Si le formateur met l'accent sur l'importance d'éclaircir ses pratiques par des apports théoriques, il peut stimuler la compréhension des situations d'enseignement et la construction de savoirs utiles à l'action professionnelle (Beckers, 2007). L'auteure affirme fermement la nécessité de mettre les savoirs et les outils cognitifs au service de l'action et de sa conceptualisation. Elle met toutefois en garde contre la pure reproduction des savoirs et invite le formateur à encourager une construction identitaire émancipatoire en s'adaptant au cheminement professionnel du futur enseignant. Ces encouragements à l'émancipation nécessitent des échanges. À cet égard, les discussions des formateurs avec le stagiaire représentent de véritables occasions pour une articulation entre terreau expérientiel et apports théoriques puisés dans les sciences de l'éducation (Brouillet et Deaudelin, 1994; Lane, 2008; Pasche Gossin, 2012).

Portelance et al. (2008) décrivent l'enseignant associé comme le formateur qui «s'intéresse aux savoirs que le stagiaire a acquis dans le milieu universitaire et l'incite à faire des liens entre ces savoirs formalisés, sa pratique et ses savoirs expérientiels» (p. 93). Le superviseur est vu comme le formateur qui « incite à une reconstruction [...] de connaissances théoriques afin de les rendre plus significatives et plus utiles en contexte d'enseignement » (p. 102). Les auteurs poursuivent : «à partir des savoirs expérientiels formulés par les stagiaires, le superviseur [...] incite le stagiaire à établir des liens entre les savoirs formels et les savoirs expérientiels et à les verbaliser» (p. 102). Ces extraits indiquent la complémentarité des rôles des deux formateurs au regard du soutien à l'articulation entre théorie et pratique, laquelle est essentielle à l'analyse critique de la pratique professionnelle.

Plusieurs auteurs s'intéressent à l'analyse réflexive de la pratique. Thornley, Parker, Read et Eason (2004) mettent en valeur le recours aux savoirs formellement reconnus pour examiner la pratique. Une attitude réflexive favoriserait le développement de l'autonomie professionnelle, fortifierait 
la construction identitaire et la prise de conscience de la singularité professionnelle (Perrenoud, 2001b). Pasche Gossin (2012) déclare que l'expérience du stagiaire est réellement formatrice s'il a l'occasion d'affronter des difficultés, de prendre une situation problème comme objet de réflexion et de transformer son expérience en la reliant à des savoirs. L'auteure se penche sur l'activité des deux formateurs du stagiaire, le formateur de la Haute école pédagogique et le formateur en établissement, en situation d'interaction avec des formés. Les résultats de sa recherche mettent en évidence trois registres d'activités des formateurs dont le registre « former ». Ce registre est réparti en trois dimensions : l'aide au retour et à la verbalisation de l'agir professionnel, l'entrainement à la mise en action des savoirs et l'élargissement des actions professionnelles. Chacune des dimensions est illustrée par des stratégies du formateur.

Pour analyser le discours des stagiaires, nous avons utilisé les stratégies de conduite du formateur regroupées dans le registre «former» de Pasche Gossin (2012). Nous avons sélectionné celles qui semblent les plus susceptibles de soutenir chez le stagiaire l'articulation des liens entre les savoirs théoriques et pratiques.

Les stratégies sélectionnées se déclinent ainsi :

- mettre en œuvre un processus de conscientisation : amener le stagiaire à prendre conscience des savoirs qui se cachent dans son agir professionnel;

- mettre en œuvre un processus d'étayage :

- amener le formé à mobiliser les concepts lui permettant de comprendre son agir professionnel;

- inciter à verbaliser les savoirs qui fondent son agir professionnel;

- inciter le formé à convoquer des concepts pour faire surgir des pistes d'actions concrètes et opérationnelles;

- favoriser une écriture réflexive qui rattache le savoir d'action à des savoirs théoriques et suscite l'émergence de pistes d'intervention.

Les précisions méthodologiques sont mentionnées dans les pages suivantes.

\section{Repères méthodologiques}

Une méthodologie de recherche de nature qualitative interprétative a permis de mieux connaitre la perception des stagiaires participants quant au soutien de leurs formateurs à l'articulation entre théorie et pratique. Cette approche méthodologique donne la possibilité de se rapprocher du monde intérieur des sujets (Karsenti et Savoie-Zajc, 2000). Elle permet de générer et traiter des données descriptives en provenance du discours des participants (Taylor et Bogdan, 1984).

\section{Les participants}

En 2012, à la demande de la chercheure principale, trois superviseures de stage ont accepté de participer à la recherche. Elles ont ensuite sollicité la participation des enseignants associés et des stagiaires avec qui elles composaient des triades. Six triades ont été constituées. Une superviseure était professeure 
à l'université. Les deux autres étaient chargées de cours dont l'une doctorante en éducation et l'autre titulaire d'une maîtrise en éducation. Toutes avaient au moins cinq ans d'expérience en enseignement. Les enseignants associés avaient pour leur part entre 15 et 25 ans d'expérience en enseignement. Ils avaient tous déjà encadré au moins trois stagiaires. Dans le cadre de leur stage d'internat du baccalauréat en enseignement secondaire de l'Université du Québec à Trois-Rivières, les stagiaires enseignaient les disciplines rattachées à leur profil de formation, français, mathématiques, univers social ou science et technologie, à des élèves de différents degrés scolaires. Ce stage a une durée de douze semaines au cours desquelles le stagiaire prend en charge la tâche complète de l'enseignant associé.

\section{La collecte des données}

Nous présentons uniquement la démarche méthodologique qui concerne l'objet du texte. Vers la fin du stage, les six stagiaires ont été rencontrés individuellement au cours d'un entretien semi-dirigé qui portait principalement sur le soutien de leurs formateurs à l'articulation entre théorie et pratique, et ce, au sujet des enseignants associés et des superviseurs de leurs quatre stages de formation à l'enseignement. Les entretiens, d'une durée minimale d'une heure, ont eu lieu à l'école du stage et ont été enregistrés sur un support numérique.

\section{Le traitement et l'analyse des données}

Les propos des stagiaires ont été transcrits intégralement. Le vocabulaire utilisé dans les opérations de codage et d'analyse des données a été préétabli à partir des stratégies de conduite du formateur, énoncées par Pasche Gossin (2012) et déjà présentées dans ce texte. Le modèle d'analyse de contenu de L'Écuyer (1990) a été choisi pour faire ressortir des éléments permettant de mieux comprendre le sens des propos des participants. Ainsi, après une première lecture flottante, nous avons établi des unités de sens rattachées aux stratégies des formateurs (Pasche Gossin, 2012) et défini des catégories. Une deuxième lecture a précédé la catégorisation des énoncés du discours des stagiaires et l'association de codes aux énoncés sélectionnés. Un travail conjoint de la chercheuse et d'une assistante de recherche, coauteures du présent texte, a permis de s'entendre sur les unités de sens, de préciser le codage (Mukamurera, Lacourse et Couturier, 2006) et de regrouper des unités de sens pour mieux rendre compte des perceptions des stagiaires. Des synthèses ont contribué à l'approfondissement de la compréhension du discours et à la structuration de la présentation des résultats. Nous avons regroupé les stratégies qui correspondaient au soutien des formateurs, tel que décrit par les stagiaires, en trois parties : soutien au rappel des savoirs théoriques qui fondent l'agir en situation, soutien à l'intervention fondée sur des savoirs théoriques, soutien à la communication écrite de la pensée. Un dernier regroupement a été créé pour rendre compte de la responsabilisation des stagiaires quant à l'articulation entre la théorie et la pratique.

L'analyse des résultats est présentée dans la section suivante. 


\section{Résultats}

L'analyse des propos des stagiaires est articulée autour de trois pôles : le rappel des savoirs théoriques qui sous-tendent l'agir professionnel, l'intervention fondée sur ces savoirs et la communication écrite de la pensée. Rappelons que les propos des stagiaires réfèrent au soutien de leurs formateurs des quatre stages de leur formation.

\section{Le soutien au rappel des savoirs théoriques qui fondent l'agir en situation}

Le soutien des formateurs au rappel des savoirs théoriques qui fondent l'agir en situation se décline ainsi : conscientiser aux savoirs théoriques qui fondent l'agir, inciter à mobiliser des concepts associés à des situations concrètes et amener à verbaliser des savoirs théoriques.

\section{Conscientiser aux savoirs théoriques qui fondent l'agir}

Dans le regard des stagiaires, le soutien des formateurs à l'articulation entre théorie et pratique évoque notamment la prise de conscience. Selon eux, les superviseurs les inciteraient à la prise de conscience de leurs savoirs théoriques. Cette stratégie conduirait les formateurs universitaires à positionner leurs exigences à l'égard des étudiants et à permettre aux futurs enseignants de se rappeler des concepts, des théories qui se cachent derrière leurs pratiques. Marie-Pier donne l'exemple suivant: "Au sujet de la théorie de la motivation, j'ai eu un superviseur qui m'avait demandé d'y revenir en allant chercher des auteurs puis de faire le lien avec ce qui se produit en classe. [...] Leur aide nous amène vraiment à réfléchir sur des évènements, comment on va s'y prendre, s'ajuster, pourquoi » $\left(\mathrm{CSUmp}^{1}\right)$. Ce soutien à la prise de conscience des savoirs théoriques susceptibles de fonder les actes professionnels peut laisser croire que les stagiaires ont besoin d'aide pour revoir des apprentissages réalisés à l'université. Cette aide se manifeste surtout par des demandes spécifiques, comme relire certains textes. Les superviseurs encourageraient les stagiaires à ramener dans leur mémoire vive des ressources emmagasinées sans être forcément accessibles.

Les stagiaires nont pas été loquaces par rapport au rôle de conscientisation exercé par les enseignants associés. Ils reçoivent des messages de leur part concernant l'importance de faire des liens entre théorie et pratique, mais peu de stratégies de soutien clairement associées à ces messages. Élodie en parle dans ces termes : "Leur soutien à l'articulation, je le remarque peu. J'entends que c'est important de faire des liens dans mes planifications, mais les enseignantes associées ne demandent rien " (CEAel). Édith corrobore en disant : "Jamais un enseignant associé ne m’a demandé d'aller vérifier dans la théorie » (CEAed).

\section{Inciter à mobiliser des concepts associés à des situations concrètes}

Il semblerait que les deux formateurs insistent sur l'importance de mobiliser des stratégies d'enseignement au moment de la planification des situations d'apprentissage et d'évaluation (SAÉ). C'est ce qu'explique Élodie : "Les enseignants associés et les superviseurs insistent beaucoup sur la conception des $S A E ́$ et sur les stratégies pédagogiques à utiliser pour les construire. C'est vraiment apporté de deux façons différentes, mais j'ai l'impression que ça se complète vraiment. J'arrive à voir un lien pertinent. " (MSUelMEAel). Cet extrait pourrait indiquer que l'insistance des formateurs est dirigée vers l'intégration de stratégies pédagogiques apprises à l'université. Selon Élodie, les formateurs lui posent des questions 
pour l'exhorter à utiliser consciemment des stratégies de planification. Ils demandent, par exemple, comment elle va ouvrir et clôturer son cours, comment elle va revenir sur ce qu'elle a enseigné.

Or, comme le soutiennent les six stagiaires, ce sont les superviseurs qui s'attendent à ce que des référents théoriques figurent dans une planification détaillée. Édith déclare que, pour répondre aux exigences des superviseurs, elle doit " travailler dans les notes en didactique pour documenter et justifier les actions de l'enseignant, des élèves : pourquoi chacun pose telle action dans la planification ». Elle apprécie l'insistance $\mathrm{du}$ superviseur : "Ça m'aide parce que ça me force à aller voir dans les documents, faire un gros travail. Je vais me référer à mes notes, je fais des lectures rapides, je vois tout ce qu'il y a à voir, je me rappelle ce que je peux faire » (MSUed). Mahina partage cet avis et ajoute que les superviseurs lui conseillent plusieurs ouvrages de référence sur les stratégies de lecture et sur la gestion éducative de la classe.

Selon les propos des stagiaires, ce sont les enseignants associés qui les incitent davantage à anticiper des difficultés ou à revenir sur des problèmes d'enseignement. Les demandes d'anticipation et de retour sur l'action peuvent pousser les stagiaires à mobiliser des concepts, mais ce soutien demeure implicite ou encore laissé à d'autres intervenants. Les paroles de Mathias sont éclairantes à ce sujet : «Je ne peux pas vraiment dire qu'ils miont aidé à faire des liens avec la théorie et ce que j'ai vu à l'université. Lorsqu'il y avait nécessité de revenir sur la théorie ou des lectures, mes enseignants associés me proposaient de chercher de l'aide parmi les intervenants de l'école, comme le psychoéducateur" (NMEAmat $\left.{ }^{2}\right)$. Édith observe que des enseignants associés ont un rapport négatif à la formation initiale : "De façon générale, quand on parle de cours de pédagogie, ce n'est pas de bons souvenirs pour les enseignants associés. Alors, c'est rare qu’ils m’invitent à intégrer cette théorie-là à la pratique. On va pas mal plus apprendre sur le tas» (NMEAed). Ce rapport négatif à la formation universitaire pourrait expliquer ce que maintiennent Mahina et Mathias : les enseignants associés ne semblent pas posséder et utiliser des ouvrages de référence sur la pédagogie et la didactique.

\section{Amener à verbaliser des savoirs théoriques qui fondent l'agir}

D'après les stagiaires, le rôle de soutien à la verbalisation des savoirs théoriques relève des superviseurs. Ces derniers leur permettraient de voir et de se représenter des situations concrètes à la lumière de théories en identifiant leurs auteurs. Unanimement, les stagiaires mentionnent que la plupart des superviseurs induisent une certaine vigilance quant à la consultation d'écrits scientifiques en sciences de l'éducation. Les stagiaires arriveraient par la suite à exprimer oralement les liens qu'ils tissent entre les contenus des écrits et les situations concrètes. C'est ce qu'avance Mathias : "Pour les superviseurs, tout ce que je dis doit avoir un lien avec quelque chose qui est théorique. Donc, je dois toujours porter attention aux théories, surveiller pour faire des liens " (VSUmat). Consulter les écrits théoriques semble être un impératif, comme en témoigne Édith : " $A ̀$ la demande des superviseurs, on a à identifier en classe un problème et après on doit parler de notre analyse théorique à l'aide de ce quion a trouvé dans la théorie et dans nos notes de cours" (VSUed). La stagiaire affirme aussi que ses notes de cours ont été utiles pour argumenter ses raisons d'agir.

Aux dires des stagiaires, le soutien des enseignants associés à la verbalisation des savoirs théoriques demeure tacite. Lors des rencontres post-observation, les enseignants associés émettraient des commentaires et formuleraient des questions ayant pour but de faire nommer les raisons d'agir en classe, comme le souligne Lauriane: "Leur soutien, c'est qu'ils viennent mobserver. Quand ils me parlent de leurs observations, ils me questionnent; ça m’aide à expliquer, à faire des liens [entre théorie et 
pratique] »(VEAla). En fait, les enseignants associés ne demanderaient pas clairement aux stagiaires de nommer des savoirs théoriques et ne questionneraient pas les stagiaires pour qu'ils les verbalisent. Par contre, c'est en semant des doutes dans l'esprit des stagiaires et en remettant en question leur agir que les enseignants associés font émerger leur bagage de savoirs théoriques ou encore leur font ressentir le besoin de fouiller, d'aller plus loin. Élodie l'explique en disant que les enseignants associés la déstabilisent et piquent sa curiosité quand ils formulent des questions comme : "T'imagines ce qui va arriver si tu t'y prends de cette façon? Que va-t-il se passer si tu fais telle chose? " (VEAel). Ainsi, ils ramènent indirectement le stagiaire à verbaliser des concepts qui peuvent expliquer ou justifier son agir professionnel.

\section{Le soutien à l'intervention fondée sur des savoirs théoriques}

L'analyse des propos des stagiaires amène également à comprendre que le soutien des formateurs à l'articulation entre théorie et pratique consiste à inciter le futur enseignant à utiliser son répertoire de savoirs théoriques pour faire surgir des pistes d'action. Les stagiaires mentionnent plus fréquemment les interventions du superviseur que celles de l'enseignant associé.

\section{Inviter à convoquer des savoirs théoriques pour faire surgir des pistes d'action}

Les stagiaires disent qu'ils sont incités à utiliser le répertoire de savoirs de leurs formateurs. Mahina apprécie particulièrement que ses formateurs lui donnent des exemples concrets des liens qu'ils font entre théorie et pratique: "Par rapport aux stratégies de lecture à intégrer aux sciences. Le superviseur avait déjà essayé ça. En fait, ceétait les stratégies de lecture, mais en univers social. Il les avait expérimentées et ensuite il a pu me dire comment ça sétait passé quand il les avait intégrées sur le terrain " (SSUmah). Si les superviseurs exposent parfois leur répertoire de savoirs théoriques pour faire surgir des pistes d'action chez les stagiaires, les enseignants associés le font rarement.

Les stagiaires affirment se retrouver devant un obstacle majeur à l'articulation entre théorie et pratique : une parcelle des savoirs théoriques des enseignants associés leur sont accessibles. Édith affirme : «Les enseignants associés sont des gens d'expérience, mais qui ont terminé leurs études depuis longtemps. [...] Ils ne sont pas au courant de ce que les stagiaires apprennent [à l'université]. [...] Ils ne sont pas à jour concernant les derniers développements et les nouvelles stratégies pédagogiques. Ils sont un peu dépassés par ça. Ils comptent sur nous pour se mettre à jour. Nous sommes donc laissés à nous-mêmes » (NSEAed). Mathias corrobore les propos d'Édith et ajoute que les enseignants associés ne sont pas à l'affut des connaissances issues de la recherche en éducation. Même s'ils participent à des formations continues, ils n'arrivent pas à fournir un apport théorique aux stagiaires.

\section{Le soutien à la communication écrite de la pensée}

Les superviseurs favorisent la communication écrite de la pensée. Ils exigent de décrire l'articulation entre théorie et pratique dans l'écriture réflexive. Bien que cette exigence représente une importante charge de travail pour les stagiaires, ces derniers la jugent tous positive et aidante. "On chiale souvent de ça. Des fois, on a l'impression de pelleter des nuages, mais oui, les travaux de stage, c'est réellement ça qui nous permet de faire des liens entre théorie et pratique », affirme Lauriane (ÉSUla). Les stagiaires sont 
amenés à écrire des journaux de bord dans lesquels ils notent régulièrement les manifestations de leurs compétences professionnelles. Ils y relèvent des faits observables analysés et interprétés à l'aide de la théorie. Élodie déclare: "C'est un temps d'arrêt durant lequel on écrit ce quion a fait, comment les élèves ont réagi, les liens avec la théorie et quelle compétence professionnelle ça touche. Ça permet de tout remettre en perspective » (ÉSUel).

Les superviseurs demandent également aux stagiaires d'analyser des incidents critiques. Être convié à l'analyse d'un incident critique permet à Mathias de relater des situations vécues en argumentant ses raisons d'agir. Il dit: "Après ça, bon, j'ai bien fait, oui ou non? Qu'est-ce que j'aurais pu faire ou qu'est-ce que je ferais différemment si l'incident se reproduisait. [...] J'ai décidé de faire ça parce que... Puis ensuite, bon, sur quelle théorie je me suis fié pour faire cette action-là " (ÉSUmat). Les stagiaires peuvent envoyer les analyses d'incidents critiques par courriel. Les superviseurs alimentent alors les stagiaires en proposant des pistes de réflexion qui reposent notamment sur des aspects théoriques ou en suggérant des références bibliographiques.

Les stagiaires parlent aussi d'un travail écrit sous forme de projet d'innovation pédagogique. Comme l'évoquent Marie-Pier et Édith, c'est un incitatif à faire de la recherche sur des pratiques pédagogiques en éducation qui ne sont pas présentes dans le milieu. Édith a créé un projet d'autoévaluation : «Pour cela, je suis allée retravailler mes notes de didactique pour documenter tout ce que l'élève et moi allions faire et pourquoi poser de telles actions " (ÉSUed).

Outre les planifications écrites, demandées et lues par les deux formateurs, il semblerait que les travaux de stage soient exclusivement demandés et lus par les superviseurs. Le soutien à la communication écrite de la pensée relèverait presque exclusivement du superviseur qui, par ailleurs, laisse beaucoup de latitude au stagiaire.

\section{La responsabilisation du stagiaire quant à l'articulation entre la théorie et pratique}

Pour Mathias, il lui revient de se rappeler que ses pratiques doivent être en lien avec «les théories quion a apprises en pédagogie » $\left(\mathrm{RS}_{\mathrm{mat}}{ }^{3}\right)$. Dans une démarche individuelle, ce stagiaire s'engage à établir des liens entre ce qu'il a appris à l'université et sa pratique ou à chercher de manière autonome s'il n'est pas en mesure de les établir dans l'immédiat. Cette responsabilisation peut contribuer au développement de l'autonomie professionnelle. Élodie doute de sa volonté à cet égard puisquelle n'a pas encore été tentée de tisser elle-même des liens entre théorie et pratique: "Peut-être que je serais mieux outillée pour gérer ma classe si j'avais plus d'informations sur l'adolescence. [...] Puis là je me dis, ça aurait été bien de chercher, de lire. Je pourrais le faire, je ne dis pas que je ne le ferai jamais, mais je ne l'ai pas fait " (NRSel). Le cas d'Élodie n'est pas isolé. Édith persiste à croire que « c'est le stagiaire qui établit les liens. Les enseignants associés et les superviseurs donnent ce qu'ils ont à donner avec leur expérience et leur bagage, mais tu fais le lien. [...] Ce ne sont pas tous les stagiaires qui creusent, mais les formateurs continuent de le demander et ils ne peuvent rien faire de plus que le demander " (RSed). 


\section{Interprétation compréhensive}

Dans cette partie du texte, nous interprétons les principaux constats qui se dégagent du point de vue des stagiaires. Nous nous attardons au soutien respectif des deux formateurs, à leur soutien conjoint et aux effets entrainés par un certain défaut de soutien de la part des formateurs.

\section{Le soutien du superviseur : un vade-mecum pour le stagiaire}

Par ses stratégies de soutien à l'articulation entre théorie et pratique, le superviseur peut représenter un vade-mecum pour les stagiaires. En effet, son soutien s'actualise dans un rappel des savoirs théoriques qui fondent l'agir en situation, que ce soit quant à la prise de conscience des savoirs théoriques, la mobilisation de concepts associés à des situations concrètes ou la verbalisation des savoirs théoriques. La conduite du superviseur vient possiblement combler le besoin tacite des stagiaires d'utiliser les savoirs théoriques, notamment les savoirs abordés à l'université. Le superviseur encadre alors une lecture expérientielle et critique des référents théoriques de la formation à l'enseignement (Altet, 2012). À cet égard, l'invitation au dialogue réflexif (Pasche Gossin, 2012) serait une stratégie souhaitable du superviseur, susceptible de soutenir l'articulation entre théorie et pratique.

Le superviseur soutient principalement l'articulation entre théorie et pratique par ses demandes relatives à l'écriture réflexive. Il importe qu'il prévienne la pure reproduction des savoirs théoriques et qu'il encourage le stagiaire à une construction identitaire émancipatoire (Beckers, 2007). Mentionnons que les travaux de stage demandés, qui nécessitent une écriture réflexive jugée chronophage par les stagiaires, sont tout de même considérés comme pertinents par ces derniers, eu égard à l'articulation entre théorie et pratique.

\section{Le soutien implicite de l'enseignant associé}

En présence du stagiaire, l'enseignant associé préconise l'articulation entre théorie et pratique, mais il semble ignorer comment guider l'enseignant apprenti dans cette direction puisque son soutien passe relativement inaperçu. Il donne pourtant lieu à un rappel tacite des savoirs théoriques qui fondent l'agir en situation. Même s'il ne demande pas de tisser des liens entre théorie et pratique, les stagiaires ont tendance à argumenter leurs choix didactiques par la verbalisation de savoirs théoriques.

L'enseignant associé aide le stagiaire à anticiper des difficultés et à faire un retour sur son enseignement. Il encourage les stagiaires à s'inspirer de son répertoire de savoirs expérientiels et leur propose des pistes d'intervention qu'il a déjà expérimentées. Il n'insiste pas sur la convocation de savoirs théoriques pour orienter les interventions. Or, de nombreuses situations rapportées par les participants à cette recherche nécessiteraient un rappel et une utilisation de savoirs théoriques. Toutefois, il semble que c'est aux stagiaires de convoquer leurs savoirs et d'en faire part à leur formateur. Aucune demande de l'enseignant associé ne leur est faite à cet égard.

À sa décharge, mentionnons que l'enseignant associé évolue dans un contexte de travail éloigné de l'université, des chercheurs et des écrits scientifiques. Avec un bagage renouvelé de connaissances en sciences de l'éducation (Schneuwly, 2012), il pourrait favoriser l'adoption de pratiques pédagogiques et 
didactiques qui s'appuient, non seulement sur l'expérience, mais aussi sur des connaissances théoriques (Beckers, 2007).

\section{Un soutien singulier, non coordonné avec l'autre formateur, mais complémentaire}

À bien des égards, l'enseignant associé et le superviseur agissent différemment et de manière indépendante. L'enseignant associé reste centré sur ses élèves. La double responsabilité qui lui incombe rend plus complexe son rôle de soutien au stagiaire quant à l'articulation entre théorie et pratique. Contrairement au formateur de terrain, le superviseur n'a pas la possibilité d'offrir au stagiaire un soutien continu à la mise en relation des savoirs expérientiels et théoriques. Or, la responsabilité de l'articulation entre théorie et pratique semble reposer en grande partie entre ses mains. Cette réalité pourrait correspondre à un effet de contrat tacite entre les deux formateurs (Portelance et Caron, 2016).

Une certaine complémentarité est palpable dans les stratégies de soutien déployées par les deux formateurs. Celle-ci est surtout perceptible dans leurs interventions au sujet de la planification de l'enseignement. Le formateur de terrain insiste sur les stratégies pédagogiques, sachant quelles ont été enseignées à l'université. Le superviseur met l'accent sur la justification des choix à la lumière d'écrits scientifiques. Les deux invitent les stagiaires, implicitement ou non, à relever le défi du décloisonnement des savoirs théoriques et pratiques de leur formation.

Un constat étonne. Les formateurs ne semblent pas encourager le débat réflexif qui permettrait au stagiaire de discuter, d'argumenter et de porter un jugement professionnel sur ses pratiques en utilisant des savoirs théoriques (Pasche Gossin, 2012). La discussion serait pourtant un moyen de tisser des liens entre savoirs théoriques et pratiques et surtout de mettre à l'épreuve les référents théoriques.

Les stagiaires ne parlent pas des rencontres en triade. Ce résultat parait surprenant. En effet, les enregistrements sonores des conversations en triade nous avaient permis de détecter un soutien clair du superviseur à l'articulation entre théorie et pratique ainsi que l'appui de l'enseignant associé qui développait les propos du superviseur. Les stagiaires percevraient-ils davantage cette complémentarité si les deux formateurs collaboraient, travaillaient et réfléchissaient ensemble à l'élaboration d'un cadre d'entretien de rétroaction susceptible de consolider la compréhension des liens entre théorie et pratique?

\section{Le défaut de soutien et ses effets sur le stagiaire}

Malgré le soutien déployé par les formateurs, les stagiaires se calculent en partie responsables de l'articulation entre théorie et pratique. Cette situation semble logique, si l'on considère, d'une part, que la responsabilité du soutien revient principalement au superviseur avec qui, par ailleurs, le stagiaire a des contacts limités et, d'autre part, que l'alternance est peu intégrative dans les faits.

Possiblement à leur insu, les formateurs encouragent le développement de l'autonomie professionnelle et la responsabilisation des stagiaires quant à l'établissement de liens entre savoirs théoriques et pratiques. Comme les stagiaires ne sont pas tous enclins à consulter des écrits et que certains sont mal outillés pour adopter une posture critique face aux savoirs véhiculés, qu'ils soient théoriques ou pratiques, le soutien des formateurs à l'articulation des liens entre la pratique et la théorie demeure essentiel. 


\section{Conclusion}

Dans la perception des stagiaires, le rapport de fonctionnalité réciproque entre la théorie et la pratique se manifeste plus faiblement chez l'enseignant associé dans l'exercice de son rôle. Le superviseur assumerait davantage la responsabilité de soutenir le stagiaire dans l'établissement de liens entre les connaissances théoriques sur l'apprentissage et l'enseignement et ses expériences professionnelles. Sur ce plan, les formateurs travaillent indépendamment l'un de l'autre, mais en complémentarité. Leurs priorités diffèrent, influencées notamment par les contextes de travail. Comment, dans un contexte de formation où l'alternance n'est pas réellement intégrative, viser une interdépendance entre savoirs théoriques et expérientiels et un soutien conjoint des deux formateurs? Une communauté de pratique rassemblant des enseignants associés et des superviseurs permettrait d'améliorer la situation.

Le nombre restreint de participants a limité notre matériel empirique et, par le fait même, la portée de notre interprétation. Recouper les données collectées auprès des stagiaires à l'aide des perceptions des enseignants associés et des superviseurs aurait été un atout. Il serait également pertinent de se pencher sur les moyens d'offrir aux stagiaires un soutien conjoint de ses formateurs à l'articulation entre théorie et pratique.

\section{Notes}

1 Les lettres majuscules représentent la stratégie de soutien du formateur et les minuscules le prénom fictif du stagiaire. Par exemple, CSU signifie la stratégie de conscientisation du superviseur et CEA celle de l'enseignant associé; mp le prénom de la stagiaire.

2 Un $\mathrm{N}$ au début du code signifie une absence de stratégie de soutien du formateur.

3 RS représente la responsabilisation du stagiaire

* Une aide financière du Conseil de recherches en sciences humaines du Canada a été accordée pour cette recherche.

\section{Références}

Altet, M. (2010). La relation dialectique entre pratique et théorie dans une formation professionnalisante des enseignants en IUFM : d'une opposition à une nécessaire articulation. Education Sciences E̋ Society, 1(1), 117-141. Repéré à http://riviste.unimc.it/index.php/es s/article/view/48

Altet, M. (2012). Comment articuler Formation et Recherche : l'exemple des apports des recherches du Réseau OPEN sur les pratiques enseignantes à la formation des enseignants. Formation et pratiques d'enseignement en questions, (14), 3745. Repéré à http://www.revuedeshep.ch/site-fpeq-n/Site FPEQ/14 files/03 altet.pdf

Altet, M., Paquay, L. et Perrenoud, P. (2002). La professionnalisation des formateurs d'enseignants : réalité émergente ou fantasme? Dans M. Altet, L. Paquay et P. Perrenoud (dir.), Formateurs d'enseignants : quelle professionnalisation? (p. 261-274). Bruxelles : De Boeck.

Beckers, J. (2007). Compétences et identité professionnelles. L'enseignement et autres métiers de l'interaction humaine. Bruxelles : De Boeck. http://dx.doi.org/10.3917/dbu.becke.2007.01

Borges, C. (2006). La formation des enseignants : entre la pratique et la formation à la pratique. Dans A. Akkari et S. Heer (dir.), La pédagogie de l'alternance dans la formation des enseignants : perspectives de recherche comparative (Actes de recherche, 5, p. 37-55).

Brien, R. (1997). Science cognitive et formation (3éd.). Québec, QC : Presses de l'Université du Québec. 
Brouillet, M.-I. et Deaudelin, C. (1994). Étude écosystémique d'un entretien de supervision de stage. Revue des sciences de l'éducation, 20(3), 443-466. http://dx.doi.org/10.7202/031736ar

Chaubet, P. et Gervais, C. (2014). Analyser l'alternance d'enquêtes entre pratique et théorie pour mieux les provoquer? Éducation et francophonie, 42(1), 151-168. http://dx.doi.org/10.7202/1024570ar

Desbiens, J.-F., Borges, C. et Spallanzani, C. (2012). J'ai mal à mon stage. Problèmes et enjeux de la formation pratique en enseignement. 21 cas étudiés. Québec, QC : Presses de l'Université du Québec.

Grossman, P. L., Smagorinsky, P. et Valencia, S. (1999). Appropriating tools for teaching English: A theoretical framework for research on learning to teach. American Journal of Education, 108(1), 1-29. http://dx.doi.org/10.1086/444230

Jacques, A. (2007). Développement de l'expertise chez les superviseurs de stage en formation à l'enseignement (Mémoire de maîtrise, Université du Québec à Montréal). Repéré à http://www.archipel.uqam.ca/723/1/M10144.pdf

Johnson, I. L. et Napper-Owen, G. (2011). The importance of role perceptions in the student teaching triad. Physical Educator, 68(1), 44-56.

Karsenti, T. et Savoie-Zajc, L. (2000). Introduction : vers une formation globale à la recherche. Dans T. Karsenti et L. Savoie-Zajc (dir.), Introduction à la recherche en éducation (p. 7-14). Sherbrooke, QC : CRP.

Lane, J. (2008). Accompagnement réflexif et raisonnement pédagogique : conceptions et pratiques de personnes enseignantes associées et manifestations du raisonnement pédagogique de leur stagiaire (Thèse de doctorat, Université de Sherbrooke). Repéré à http://savoirs.usherbrooke.ca/handle/11143/941

L'Écuyer, R. (1990). Méthodologie de l'analyse développementale des contenus. Méthode GPS et concept de soi. Québec, QC : Presses de l'Université du Québec.

L'Hostie, M., Monney, N. et Nadeau-Tremblay, S. (2013). Une recherche-action-formation en communauté de pratique. Un projet novateur pour la formation continue des enseignants associés. Dans C. Landry et C. Garant (dir.), Formation continue, recherche et partenariat (p. 63-91). Québec, QC : Presses de l'Université du Québec.

Malglaive, G. (1994). Alternance et compétences. Cahiers pédagogiques, 320, $26-28$.

Malglaive, G. et Weber, A. (1982). Théorie et pratique, approche critique de l'alternance en pédagogie. Revue française de pédagogie, 61(1), 17-27. http://dx.doi.org/10.3406/rfp.1982.1861

Malo, A. (2000). Savoirs de formation et savoir d'expérience : un processus de transformation. Éducation et francophonie, 28(2), 216-235. Repéré à http://www.acelf.ca/c/revue/pdf/EF-XXVIIIno2-Savoirs-de-formation-et-savoir-d-experience.pdf

Malo, A. (2005). Parcours d'un stagiaire en enseignement secondaire : adhésion ou déviance par rapport à des savoirs proposés. Dans C. Gervais et L. Portelance (dir.), Des savoirs au cœur de la profession enseignante : contextes de construction et modalités de partage (p. 65-84). Sherbrooke, QC : CRP.

Merhan, F., Ronveaux, C. et Vanhulle, S. (dir.) (2007). Alternances en formation. Bruxelles : De Boeck. http://dx.doi.org/10.3917/dbu.merha.2007.01

Ministère de l'Éducation du Québec. (2001). La formation à l'enseignement. Les orientations, les compétences professionnelles. Québec, QC : Gouvernement du Québec. Repéré à http://www.education.gouv.qc.ca/fileadmin/site web/ documents/reseau/formation titularisation/formation enseignement orientations EN.pdf

Mukamurera, J., Lacourse, F. et Couturier, Y. (2006). Des avancées en analyse qualitative : pour une transparence et une systématisation des pratiques. Recherches qualitatives, 26(1), 110-138. Repéré à http://www.recherche-qualitative. qc.ca/documents/files/revue/edition_reguliere/numero26(1)/mukamurera al ch.pdf

Pasche Gossin, F. M. (2012). Agir en formateur dans une formation des enseignants par alternance : une analyse de l'activité des formateurs dans trois dispositifs de formation par alternance (Thèse de doctorat, Université de Genève). Repéré à https://archive-ouverte.unige.ch/unige: 24167

Pentecouteau, H. (2012). L'alternance dans une formation professionnelle universitaire. De l'idéal épistémologique aux contradictions pédagogiques. Revue internationale de pédagogie de l’enseignement supérieur, 28(1), 1-11. Repéré à https://ripes.revues.org/605 
Perrenoud, P. (2001a, avril). Les sciences de l'éducation proposent-elles des savoirs mobilisables dans l'action? Communication présentée au symposium «La place des sciences humaines et sociales dans l'expertise et les savoirs professionnels des enseignants » dans le cadre des Rencontres du Réseau Éducation et Formation (REF), Montréal, QC. Repéré à http:// www.unige.ch/fapse/SSE/teachers/perrenoud/php main/php 2001/2001 12.html

Perrenoud, P. (2001b). Articulation théorie-pratique et formation de praticiens réflexifs en alternance. Dans P. Lhez, D. Millet et B. Séguier (dir.), Alternance et complexité en formation. Éducation - Santé - Travail social (p. 10-27). Repéré à http:// www.unige.ch/fapse/SSE/teachers/perrenoud/php_main/php_2001/200132.html

Portelance, L. (2010). Analyse des perceptions du soutien d'un enseignant associé à la formation du stagiaire. Éducation et francophonie, 38(2), 21-38. http://dx.doi.org/10.7202/1002162ar

Portelance, L. et Caron, J. (2016, mai). Comment les superviseurs manifestent-ils leurs compétences au regard du développement professionnel des futurs enseignants? Point de vue de stagiaires. Communication présentée au colloque Superviser un stagiaire : quelle formation, quelles compétences pour le formateur en contexte de stage? $3^{\mathrm{e}}$ colloque du CRIFPE, Montréal, QC.

Portelance, L., Gervais, C., Lessard, M. et Beaulieu, P. et collaborateurs. (2008). La formation des enseignants associés et des superviseurs universitaires. Rapport de recherche. Cadre de référence. Repéré à https://www.usherbrooke.ca/education/ fileadmin/sites/education/documents/Intranet/Documents officiels/Rapport Cadre reference 2009.pdf

Portelance, L. et Legendre, M.-F. (2001). Les études de cas comme modalités de mise en discours de la pratique : leur contribution au développement de la compétence professionnelle des futurs enseignants. Dans A. Beauchesne et $\mathrm{S}$. Martineau (dir.), La recherche en éducation et le développement de la pratique professionnelle en enseignement (p. 17-35). Sherbrooke, QC : CRP.

Portelance, L., Martineau, S. et Caron, J. (sous presse). What collaboration by knowledge sharing between cooperating teachers and university supervisors? Brock Education Journal.

Portelance, L. et Van Nieuwenhoven, C. (2010). Fondements théoriques et méthodologies des conduites de recherche portant sur la formation professionnelle en alternance. Éducation et francophonie, 38(2), 1-5. http://dx.doi.org/10.7202/1002159ar

Schneuwly, B. (2012). Praticien réflexif, réflexion et travail enseignant : l'oubli de l'objet et des outils d'enseignement. Dans M. Tardif, C. Borges et A. Malo (dir.), Le virage réflexif en éducation. Où en sommes-nous 30 ans après Schön? (p. 73-91). Bruxelles : De Boeck. http://dx.doi.org/10.3917/dbu.tardi.2012.01.0073

Stürmer, K., Könings, K. D. et Seidel, T. (2013). Declarative knowledge and professional vision in teacher education: effect of courses in teaching and learning. British Journal of Education Studies, 83(3), 467-483.

http://dx.doi.org/10.1111/j.2044-8279.2012.02075.x

Tardif, M., Gérin-Lajoie, D., Anderson, S., Lessard, C., Gauthier, C., Mujawamariya, D., .. Lenoir, Y. (2001). Les programmes de formation initiale (FI) à l'enseignement: Bilan des réformes récentes et nouvelles tendances pour une formation professionnelle efficace. Repéré à https://depot.erudit.org/id/003348dd

Taylor, S. J. et Bogdan, R. (1984). Introduction to qualitative research methods: the search for meanings (2 ${ }^{\mathrm{e}}$ éd.). New York, NY : Wiley.

Thornley, C., Parker R., Read, K. et Eason V. (2004). Developing a research partnership: teachers as researchers and teacher educators. Teachers and Teaching: Theory and Practice, 10(1), 20-33. http://dx.doi.org/10.1080/1354060032000017089

Waege, K. et Haugalokken, O. K. (2013). Research-based and hands-on practical teacher education: An attempt to combine the two. Journal of Education for Teaching, 39(2), 235-249.

Wentzel, B. (2012). Réflexivité et formation professionnelle des enseignants : actualités d'un paradigme en construction. Dans M. Tardif, C. Borges et A. Malo (dir.), Le virage réflexif en éducation. Où en sommes-nous 30 ans après Schön? (p. 143-160). Bruxelles : De Boeck. http://dx.doi.org/10.3917/dbu.tardi.2012.01.0143

\section{Pour citer cet article}

Caron, J. et Portelance, L. (2017). Perceptions de stagiaires quant au soutien des deux formateurs à l'articulation entre théorie et pratique. Formation et profession, 25(1), 34-49. http://dx.doi.org/10.18162/fp.2017.367 